\title{
TOTALLY ANALYTIC SPACES UNDER $V=L$
}

\author{
ZOLTÁN BALOGH AND HEIKKI JUNNILA
}

\begin{abstract}
The following results obtain under the axiom of constructibility $(V=L)$ : Assume that every subset of a topological space $X$ is analytic. Then $X$ is $\sigma$-left-separated. Moreover, if the character of $X$ is $\leqslant \omega_{1}$, then $X$ is $\sigma$-discrete.

Assume that $X$ is a perfectly normal space of character $\leqslant \omega_{1}$ such that every subset of $X$ belongs to the $\sigma$-algebra generated by the analytic subsets of $X$. Then $X$ is $\sigma$-discrete.
\end{abstract}

1. Introduction. By an analytic subset of a topological space $X$, we mean a set that can be obtained from a family of closed subsets of $X$ by the $A$-operation, i.e., a set -f the form $\cup_{\phi \in \omega_{\omega}} \cap_{n \in \omega} F_{\phi \mid n}$, where the sets $F_{s}, s \in{ }^{<\omega} \omega$, are closed in $X$. We say that $X$ is a totally analytic space if each subset of $X$ is analytic. This notion generalizes that of a space in which each subset is an $F_{\sigma}$-set.

A space $X$ is $\sigma$-discrete ( $F_{\mathrm{o}}$-discrete) if $X$ is the countable union of discrete (and closed) subspaces. $X$ is left-separated if there exists a well-order $<$ on $X$ such that all the initial segments $\{y \in X: y<x\}, x \in X$, are closed in $X$. We say that $X$ is $\sigma$-left-separated if $X$ is the countable union of left-separated subspaces. Note that the concept of an $F_{\sigma}$-left-separated space is superfluous, since such a space is actually left-separated; in particular, every $F_{\sigma}$-discrete space is left-separated.

The character of a space $X$ is the least cardinal number $\kappa$ such that every point of $X$ has a neighbourhood-base consisting of at most $\kappa$ sets; the character of $X$ is denoted by $\chi(X)$.

The results in this paper give some partial answers to the question whether it is consistent with ZFC that every totally analytic space is $\sigma$-discrete. We can only aim for a consistency result since it follows from $\mathrm{MA}+\neg \mathrm{CH}$ that $\mathbf{R}$ has uncountable (hence non- $\sigma$-discrete) subspaces that are totally analytic (and in fact, with each subset an $F_{\mathrm{o}}$-set). To obtain our results, we use Gödel's constructibility axiom $V=L$. Previous to the results below, G. M. Reed has shown that, under $V=L$, each first countable normal space with every subset an $F_{\sigma}$-set is $F_{\sigma}$-discrete [8], and R. W. Hansell has shown that, under $V=L$, a totally analytic space $X$ of character $\leqslant \omega_{1}$ is $\sigma$-discrete provided that the product of $X$ with the irrationals is normal [5]. Like Reed and Hansell, we rely on W. G. Fleissner's techniques and results in [2] (dealing with the seemingly unrelated topic of collectionwise Hausdorffness of normal spaces of character $\leqslant \omega_{1}$ under $V=L$ ).

Received by the editors November 23, 1981 and, in revised form. May 25, 1982.

1980 Mathematics Subject Classification. Primary 54H05, 04A15, 28A05; Secondary 54A25. 54A35.

Key words and phrases. $V=L$, analytic set, $\sigma$-discrete, left-separated, character. 
2. The main theorems. We have only been able to obtain $\sigma$-discreteness from $V=L$ for totally analytic spaces under the restriction that the spaces under consideration have character $\leqslant \omega_{1}$; for general spaces, we have the following result:

THEOREM $2.1(V=L)$. Each totally analytic space is $\sigma$-left-separated.

The proof of Theorem 2.1 is a modification of Fleissner's proof of the main result in [2]. We shall give some lemmas from which the theorem follows; for a few of these lemmas, we do not give a proof but only indicate the changes that are needed in the proofs of the corresponding results in [2].

In [2], mappings $A \rightarrow \omega_{1}$ were used to code open separations of subsets of a discrete subset $A$ of a space $X$ (of character $\leqslant \omega_{1}$ ). Here we use mappings $X \rightarrow{ }^{\omega} \omega$ to code partitions of $X$ into analytic sets. (Note that, under $V=L,\left|{ }^{\omega} \omega\right|=\omega_{1}$.)

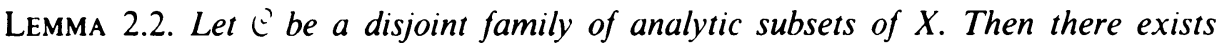
$f: X \rightarrow{ }^{\omega} \omega$ such that, for each $C \in \mathcal{E}$,

$$
C=\bigcup_{\phi \in{ }^{\omega} \omega} \bigcap_{n \in \omega} \overline{\{x \in C: f(x)|n=\phi| n\}} .
$$

Proof. For every $C \in \mathcal{E}$, let $\left\{F_{s}(C): s \in{ }^{<\omega} \omega\right\}$ be a family of closed subsets of $X$ such that $C=\cup_{\phi \in \omega_{\omega}} \cap_{n \in \omega} F_{\phi \mid n}(C)$. Define $f: X \rightarrow{ }^{\omega} \omega$ as follows. If $x \in X \sim \cup \mathcal{E}$, let $f(x)$ be an arbitrary element of ${ }^{\omega} \omega$. If $x \in C \in \mathcal{E}$, let $f(x) \in{ }^{\omega} \omega$ be such that $x \in \cap_{n \in \omega} F_{f(x) \mid n}(C)$. That $f$ has the required property follows by observing that for all $C \in \mathcal{E}, \phi \in{ }^{\omega} \omega$ and $n \in \omega,\{x \in C: f(x)|n=\phi| n\} \subset F_{\phi \mid n}(C)$.

A connection between mappings $X \rightarrow{ }^{\omega} \omega$ and $\sigma$-left-separatedness of $X$ is given in the following lemma. Call $X A$-left-separated if there exists a well-order $<$ on $X$ and a map $f: X \rightarrow{ }^{\omega} \omega$ such that, for each $x \in X$,

$$
x \notin \bigcup_{\phi \in \omega_{\omega} \omega} \bigcap_{n \in \omega} \overline{\{y<x: f(y)|n=\phi| n\}} .
$$

LEMMA 2.3. (i) Every A-left-separated space is $\sigma$-left-separated.

(ii) If a space has a countable cover by analytic left-separated subspaces then the space is A-left-separated.

Proof. (i) Assume that $X,<$ and $f$ satisfy the condition defining $A$-left-separatedness. For each $x \in X$, let $n(x) \in \omega$ be such that

$$
x \notin \overline{\{y<x: f(y)|n(x)=f(x)| n(x)\}} .
$$

For each $s \in{ }^{<\omega} \omega$, let $L_{s}=\{x \in X: f(x) \mid n(x)=s\}$. Then the sets $L_{s}, s \in{ }^{<\omega} \omega$, cover $X$, and it is easy to check that these sets are left-separated as subspaces of $X$.

(ii) Assume that $X$ has a cover $\left\{A_{k}: k<\omega\right\}$ by analytic subsets and that, for every $k<\omega$, there exists a well-ordering $<_{k}$ of $A_{k}$ such that, for each $x \in A_{k}, x$ $\notin \overline{\left\{y \in A_{k}: y<_{k} x\right\}}$ For each $k \in \omega$, let $\left\{F_{s}^{k}: s \in{ }^{<\omega} \omega\right\}$ be a family of closed subsets of $X$ such that $A_{k}=\cup_{\phi \in \omega_{\omega}} \cap_{n \in \omega} F_{\phi \mid n}^{k}$ and, for all $\phi \in{ }^{\omega} \omega$ and $n \in \omega$, $F_{\phi \mid n+1}^{k} \subset F_{\phi \mid n}^{k}$. For each $x \in X$, let $k(x)$ be the least $k \in \omega$ such that $x \in A_{k}$, and let $f(x) \in{ }^{\omega} \omega$ be such that $x \in \bigcap_{n \in \omega} F_{f(x) \mid n}^{k(x)}$. Define a well-order $\prec$ on $X$ by setting $y \prec x$ iff either $k(y)<k(x)$ or $k(y)=k(x)$ and $y<{ }_{k} x$. To show that $X$ is 
$A$-left-separated with respect to $f$ and $\prec$, let $x \in X$ and let $k=k(x)$. For all $\phi \in{ }^{\omega} \omega$ and $n \in \omega$, we have $\{y \prec x: f(y)|n=\phi| n\}=\cup_{j<k}\left\{y \in A_{j}: f(y)|n=\phi| n\right\} \cup$ $\left\{y \in A_{k}: y<_{k} x\right.$ and $\left.f(y)|n=\phi| n\right\}$, and it follows that

$$
\overline{\{y \prec x: f(y)|n=\phi| n\}} \subset \bigcup_{j<k} F_{\phi \mid n}^{j} \cup \overline{\left\{y \in A_{k}: y<_{k} x\right\}} \cap F_{\phi \mid n}^{k} .
$$

Further, since the sets $F_{\phi \mid n}^{i}, n \in \omega$, are decreasing, it follows that

$$
\begin{aligned}
\bigcap_{n \in \omega} \overline{\{y<x: f(y)|n=\phi| n\}} & \subset \bigcup_{j<k}\left(\bigcap_{n \in \omega} F_{\phi \mid n}^{j}\right) \cup \overline{\left\{y \in A_{k}: y<_{k} x\right\}} \cap \bigcap_{n \in \omega} F_{\phi \mid n}^{k} \\
& \subset \bigcup_{j<k} A_{j} \cup \overline{\left\{y \in A_{k}: y<_{k} x\right\}} \cap A_{k} \\
& =\bigcup_{j<k} A_{j} \cup\left\{y \in A_{k}: y<_{k} x\right\}=\{y \in X: y \prec x\} .
\end{aligned}
$$

Consequently, $\cup_{\phi \in \omega^{\omega} \omega} \cap_{n \in \omega} \overline{\{y \prec x: f(y)|n=\phi| n\}} \subset\{y: y \prec x\}$; since $x \notin$ $\{y: y \prec x\}$, we have completed the proof of (ii).

REMARK. By Lemma 2.3, a totally analytic space is $\sigma$-left-separated iff the space is $A$-left-separated. Thus we can prove Theorem 2.1 by showing that, under $V=L$, each totally analytic space is $A$-left-separated. Note that, corresponding to the similarly marked result on p. 295 of [2], we have the following consequence of Lemma 2.3:

(*) If a totally analytic space is the countable union of $A$-left-separated subspaces, then the space is $A$-left-separated.

For each cardinal number $\kappa$, denote by TALS $(\kappa)(\operatorname{TALS}(<\kappa))$ the statement that each totally analytic space of cardinality $\kappa$ (of cardinality $<\kappa$ ) is $A$-left-separated. For $\kappa \leqslant \omega$, TALS $(\kappa)$ holds trivially. We shall prove Theorem 2.1 by using transfinite induction on $\kappa$ to show that TALS $(\kappa)$ holds for every uncountable cardinal $\kappa$. For a singular cardinal $\kappa$ of cofinality $\omega$, it follows from $(*)$ that $\operatorname{TALS}(<\kappa) \Rightarrow \operatorname{TALS}(\kappa)$. Hence it suffices to prove that TALS $(<\kappa) \Rightarrow \operatorname{TALS}(\kappa)$ whenever $\kappa$ is either regular or singular of cofinality $>\omega$.

Without loss of generality, we may assume that the underlying set of our totally analytic space $X$ is a cardinal number $\kappa$; we shall make this assumption for the rest of the proof of Theorem 2.1 .

For all $f: \kappa \rightarrow{ }^{\omega} \omega$ and $\alpha<\kappa$, let $W_{\alpha}^{f}=\cup_{\phi \in{ }^{\omega} \omega} \bigcap_{n \in \omega} \overline{\{\beta<\alpha: f(\beta)|n=\phi| n\}}$. The following result corresponds to Lemma 1 of [2]:

LEMmA 2.4. Assume TALS $(<\kappa)$. Either $A_{f}=\left\{\alpha<\kappa: W_{\alpha}^{f} \neq \alpha\right\}$ is stationary for each $f: \kappa \rightarrow{ }^{\omega} \omega$ or $X$ is $A$-left-separated.

Proof. Assume that there exists $f: \kappa \rightarrow{ }^{\omega} \omega$ and a cub $C \subset \kappa$ such that $C \cap A_{f}=\varnothing$. For each $\alpha \in \kappa$, let $\gamma(\alpha)$ be the least element of the set $\{\gamma \in C: \alpha<\gamma\}$. For every $\gamma \in C$, it follows from TALS $(<\kappa)$ that there exists a well-order $<_{\gamma}$ on $\gamma$ and a map $f_{\gamma}: \gamma \rightarrow{ }^{\omega} \omega$ such that for each $\alpha \in \gamma, \cup_{\phi \in \omega_{\omega} \omega} \cap_{n \in \omega} \overline{\left\{\beta<_{\gamma} \alpha: f_{\gamma}(\beta)|n=\phi| n\right\}} \cap \gamma=$ $\left\{\beta \in \gamma: \beta<{ }_{\gamma} \alpha\right\}$. Define a well-order $\prec$ on $\kappa$ by setting $\alpha<\beta$ iff either $\gamma(\alpha)<\gamma(\beta)$ or $\gamma(\alpha)=\gamma(\beta)$ and $\alpha<_{\gamma(\alpha)} \beta$. Define a binary operation $\uparrow$ on ${ }^{\omega} \omega$ by the condition that $\theta \uparrow \psi(2 n)=\theta(n)$ and $\theta \uparrow \psi(2 n+1)=\psi(n)$ for all $\theta, \psi \in \in^{\omega} \omega$ and $n \in \omega$. Define 
a map $g: \kappa \rightarrow{ }^{\omega} \omega$ by setting $g(\alpha)=f \uparrow f_{\gamma(\alpha)}$ for each $\alpha \in \kappa$. We show that $X$ is $A$-left-separated with respect to $\prec$ and $g$. Let $\alpha \in \kappa$, and let $\gamma=\gamma(\alpha)$. Denote by $\delta$ the largest element of the set $\{\rho \in C \cup\{0\}: \rho \leqslant \alpha\}$, and let $B=\{\beta \in \gamma \sim \delta$ : $\left.\beta<{ }_{\gamma} \alpha\right\}$; note that $\{\beta \in \kappa: \beta<\alpha\}=\delta \cup B$ and that, for each $\beta \in B, \gamma(\beta)=\gamma$. Let $\phi \in{ }^{\omega} \omega$. To show that $\bigcap_{n \in \omega} \overline{\{\beta \prec \alpha: g(\beta)|n=\phi| n\}} \subset\{\beta: \beta \prec \alpha\}$, let $\theta \in{ }^{\omega} \omega$ and $\psi \in{ }^{\omega} \omega$ be such that $\phi=\theta \uparrow \psi$. Then, for each $n \in \omega, \quad\{\beta<\alpha: g(\beta) \mid 2 n=$ $\phi \mid 2 n\}=\left\{\beta \prec \alpha: f(\beta)|n=\theta| n\right.$ and $\left.f_{\gamma(\beta)}(\beta)|n=\psi| n\right\} \subset\{\beta \in \delta: f(\beta) \mid n=$ $\theta \mid n\} \cup\left\{\beta \in B: f(\beta)|n=\theta| n\right.$ and $\left.f_{\gamma}(\beta)|n=\psi| n\right\}$. It follows that

$$
\begin{aligned}
\bigcap_{n \in \omega} \overline{\{\beta<\alpha: g(\beta)|n=\phi| n\}} \subset \bigcap_{n \in \omega} \overline{\{\beta \in \delta: f(\beta)|n=\theta| n\}} \\
\cup\left[\bigcap_{n \in \omega} \overline{\left\{\beta \in B: f_{\gamma}(\beta)|n=\psi| n\right\}} \cap \bigcap_{n \in \omega} \overline{\{\beta \in B: f(\beta)|n=\theta| n\}}\right] ;
\end{aligned}
$$

consequently, as $\delta, \gamma \in \kappa \sim A_{f}$ and $B \subset\left\{\beta \in \gamma: \beta<_{\gamma} \alpha\right\}$,

$$
\begin{gathered}
\bigcap_{n \in \omega} \overline{\{\beta \prec \alpha: g(\beta)|n=\phi| n\}} \subset \delta \cup\left[\bigcap_{n \in \omega} \overline{\left\{\beta<_{\gamma} \alpha: f_{\gamma}(\beta)|n=\psi| n\right\}} \cap \gamma\right] \\
\subset \delta \cup\left\{\beta \in \gamma: \beta<_{\gamma} \alpha\right\}=\{\beta \in \kappa: \beta \prec \alpha\} .
\end{gathered}
$$

LEMMA $2.5(V=L)$. For a regular $\kappa, \operatorname{TALS}(<\kappa) \Rightarrow \operatorname{TALS}(\kappa)$.

Proof. If we use Lemma 2.4 above to substitute for Lemma 1 of [2], the proof of Lemma 2.5 is almost identical to that of Lemma 3 of [2]. We assume that TALS $(<\kappa)$ holds but there is a totally analytic space $X$ with underlying set $\kappa$ such that $X$ is not $A$-left-separated. Then the sets $A_{f}$ of Lemma 2.4 are all stationary. It is easily seen that for all $f, g: \kappa \rightarrow{ }^{\omega} \omega$ and $\alpha \in \kappa$, we have $A_{f} \cap(\alpha+1)=A_{g} \cap(\alpha+1)$ whenever $f|\alpha=g| \alpha$. Consequently, the sets $A_{f}$, for $f: \kappa \rightarrow^{\omega} \omega$, form what Fleissner calls a stationary system, and we can apply Lemma 2 of [2] to conclude that there exists $S \subset \kappa$ and $\Phi(\alpha): \alpha \rightarrow{ }^{\omega} \omega$, for $\alpha \in S$, such that for each $f: \kappa \rightarrow^{\omega} \omega$, the set $\{\alpha: \Phi(\alpha)=f \mid \alpha\}$ is a stationary set contained in $A_{f}$.

Whenever, $E \subset \kappa, f$ is a map $E \rightarrow{ }^{\omega} \omega$ and $B \subset E$, write

$$
f^{\#}(B)=\bigcup_{\phi \in \omega^{\omega} \omega} \bigcap_{n \in \omega} \overline{\{\beta \in B: f(\beta)|n=\phi| n\}} .
$$

The proof of Lemma 3 of [2] works here once we make the following changes: for both $U_{\alpha}^{f}$ and $\overline{U_{\alpha}^{f}}$ use the set $f^{\#}(\alpha \cap H)$; for $V_{\alpha}^{f}$ and $\overline{V_{\alpha}^{f}}$ use $f^{\#}(\alpha \cap K)$; for $W_{\alpha}^{f}$ and $\overline{W_{\alpha}^{f}}$ use $f^{\#}(\alpha)$; for the notion " $f^{\#}$ separates $H$ and $K$ " use the condition $f^{\#}(H) \cap$ $f^{\#}(K)=\varnothing$.

The changes indicated in the above proof also work to show that the results corresponding to Lemmas 4 and 5 of [2] hold in the present situation; in particular, we have the following result:

LeMma $2.6(\mathrm{GCH})$. For a singular $\kappa$ of cofinality $>\omega, \operatorname{TALS}(<\kappa) \Rightarrow \operatorname{TALS}(\kappa)$.

The above results complete the proof of Theorem 2.1. Note that since each $F_{\sigma}$-left-separated space is left-separated, we have the following consequence of Theorem 2.1. 
COROLlARY $2.7(V=L)$. If every subset of a topological space is an $F_{\mathrm{o}}$-set, then the space is left-separated.

Every uncountable left-separated space contains a nonseparable subspace; hence Theorem 2.1 gives the following result.

COROllaRY $2.8(V=L)$. Every totally analytic hereditarily separable space is countable.

In light of Theorem 2 of [1], and Theorem 1 of [1], Theorem 4 of [4] or Corollary 4.9 of [6], the following is a consequence of Theorem 2.1.

COROLlaRY $2.9(V=L)$. If a totally analytic space is either symmetrizable or semistratifiable, then the space is $F_{\sigma}$-discrete.

Our second theorem indicates one more particular case, beyond those given in the above corollary, in which the conclusion of Theorem 2.1 can be strengthened to $\sigma$-discreteness. Whereas the proof of Theorem 2.1 was a modification of Fleissner's proof of the theorem in [2], the proof below consists of an application of that theorem itself. We also use a lemma from [5] on the representation of analytic sets.

THEOREM $2.10(V=L)$. Each totally analytic space of character $\leqslant \omega_{1}$ is $\sigma$-discrete.

Proof. Let $X$ be a totally analytic space with $\chi(X) \leqslant \omega_{1}$. To show that $X$ is $\sigma$-discrete, we construct a normal space $Y$ with $\chi(Y) \leqslant \omega_{1} ; V=L$ and Fleissner's theorem imply that $Y$ is collectionwise Hausdorff, and from this we infer that $X$ is $\sigma$-discrete.

Let $\mathcal{H}$ be the filter consisting of all sets $H \subset{ }^{<\omega} \omega$ such that there exists $\phi \in{ }^{\omega} \omega$ such that for each $\psi \in{ }^{\omega} \omega$, if $\psi \geqslant \phi$, then there is $n \in \omega$ such that $\{\psi \mid m: m \geqslant n\} \subset H$ (these sets are called "final segments of ${ }^{<\omega} \omega$ " in [5]). Let $Y=X \times X \times{ }^{<\omega} \omega$. For each $L \subset X$, denote by $L^{*}$ the subset $\{\langle x, x, \varnothing\rangle: x \in L\}$ of $Y$. For all $H \in \mathbb{K}$ and $x \in X$, and for each map $g$ from $H$ into the set of all neighborhoods of $x$ in $X$, let

$$
U(x, g)=\{\langle x, x, \varnothing\rangle\} \cup(\{x\} \times X \times H) \cup \bigcup_{s \in H}(g(s) \times\{x\} \times\{s\}) .
$$

Topologize $Y$ by making the points of $Y \sim X^{*}$ isolated and by using the sets $U(x, g)$ to define a neighborhood base for $\langle x, x, \varnothing\rangle \in X^{*}$.

Since $\left.\right|^{<\omega} \omega|=\omega,| \mathcal{K} \mid \leqslant 2^{\omega}, \chi(X) \leqslant \omega_{1}$, and $2^{\omega}=\omega_{1}$ (under $V=L$ ), it is easily seen that $\chi(Y) \leqslant \omega_{1}$. To see that $Y$ is normal, note first that $X^{*}$ is a closed discrete subset of $Y$ with discrete complement. Consequently, $Y$ is normal provided that for each $L \subset X$, the sets $L^{*}$ and $(X \sim L)^{*}$ can be separated by open subsets of $Y$. Let $L \subset X$. Since $X$ is totally analytic, there are closed subsets $F_{s}$ and $K_{s}, s \in{ }^{<\omega} \omega$, of $X$ such that $L=\cup_{\phi \in \omega_{\omega}{ }} \bigcap_{n \in \omega} F_{\phi \mid n}$ and $X \sim L=\cup_{\phi \in{ }^{\omega} \omega} \cap_{n \in \omega} K_{\phi \mid n}$; by Lemma 2 of [5], we can choose the sets $F_{s}$ and $K_{s}$ so that for all $\phi, \psi \in{ }^{\omega} \omega$ and $n \in \omega$, $F_{\phi \mid n+1} \subset F_{\phi \mid n}$ and $K_{\phi \mid n+1} \subset K_{\phi \mid n}$ and, if $\phi \leqslant \psi$, then $F_{\phi \mid n} \subset F_{\psi \mid n}$ and $K_{\phi \mid n} \subset K_{\psi \mid n}$. For each $x \in X$, let $H_{x}=\left\{s \in{ }^{<\omega} \omega: x \in F_{s} \sim K_{s}\right\}$ and $J_{x}=\left\{s \in{ }^{<\omega} \omega: x \in K_{s} \sim F_{s}\right\}$; note that if $x \in L$, then $H_{x} \in \mathcal{H}$ and if $x \in X \sim L$, then $J_{x} \in \mathcal{K}$. For each $s \in{ }^{<\omega} \omega$, let $g(s)=X \sim K_{s}$ and $f(s)=X \sim F_{s}$. Let $V=\cup_{x \in L} U\left(x, g \mid H_{x}\right)$ and $W=$ $\cup_{x \in X \sim L} U\left(x, f \mid J_{x}\right)$, and note that these are open subsets of $Y$ containing $L^{*}$ and 
$(X \sim L)^{*}$, respectively. We show that $V \cap W=\varnothing$. Assume on the contrary that there are $x \in L, z \in X \sim L$ and $\langle u, v, s\rangle \in U\left(x, g \mid H_{x}\right) \cap U\left(z, f \mid J_{x}\right)$. We have $s \in H_{x} \cap J_{x}$ and it follows that $x \in F_{s}$ and $z \in K_{s}$. Note that either $u=x$ or $u=z$. If $u=x$, then $x \in f(s)=X \sim F_{s}$, and if $u=z$, then $z \in g(s)=X \sim K_{s}$; hence we have a contradiction in both cases.

By Fleissner's theorem in [2], $Y$ is collectionwise Hausdorff. Consequently, there are $H_{x} \in \mathfrak{H}, x \in X$, and for each $x \in X$, a map $g_{x}$ from $H_{x}$ to the neighborhoods of $x$ in $X$ such that for any two distinct points $x$ and $y$ of $X, U\left(x, g_{x}\right) \cap U\left(y, g_{y}\right)=\varnothing$. For each $s \in{ }^{<\omega} \omega$, let $D_{s}=\left\{x \in X: s \in H_{x}\right\}$. Then $X=\cup_{s \in{ }^{<} \omega_{\omega}} D_{s}$, and for each $s \in S$, the subspace $D_{s}$ of $X$ is discrete because, for each $x \in D_{s}$ and for any $y \in D_{s} \sim\{x\}$, we have $\langle y, x, s\rangle \in U\left(y, g_{y}\right) \sim U\left(x, g_{x}\right)$ and hence $y$ cannot belong to the neighborhood $g_{x}(s)$ of $x$.

RemarKs. $1^{\circ}$. Note that the construction used above can be used (with a slight change in the proof of normality of $Y$ ) to show that, under $V=L$, a space of character $\leqslant \omega_{1}$ is $\sigma$-discrete provided that each subset of the space is $(\hat{j}$-analytic (i.e., can be obtained by the $A$-operation from a family of open sets).

$2^{\circ}$. In a $\sigma$-discrete space, every subset is a Borel-set (more precisely, the countable union of sets, each of which is the intersection of an open set with a closed set). In spite of Theorem 2.10, the following problem remains open: Is it true under $V=L$ that a space of character $\leqslant \omega_{1}$ has to be $\sigma$-discrete if each subset of the space is a Borel-set? The one-point compactification of an uncountable discrete space provides an example of a space in which each subset is Borel while there are nonanalytic subsets (note that if $x \in X$ is such that $X \sim\{x\}$ is analytic, then $\{x\}$ is a $G_{\delta}$-set).

3. Some related results. An interesting problem motivated by the previous results is whether a metrizable space has to be $\sigma$-discrete under $V=L$ provided each subset of the space is projective. We have been unable to solve that problem even for the first level of projectivity $\left(\Sigma_{2}^{1}\right)$ beyond analyticity. A related problem is whether a metrizable space has to be $\sigma$-discrete under $V=L$ in case each subset of the space belongs to the $\sigma$-algebra generated by the analytic subsets of the space. A positive solution to the latter problem is contained in the following results.

Let us call a subset $A$ of a topological space $X$ strongly analytic if there are open subsets $G_{s}$ of $X$, for $s \in{ }^{<\omega} \omega$, such that

$$
A=\bigcup_{\phi \in \omega^{\omega} \omega} \bigcap_{n \in \omega} G_{\phi \mid n}=\bigcup_{\phi \in{ }^{\omega} \omega} \bigcap_{n \in \omega} \overline{G_{\phi \mid n}} .
$$

Each strongly analytic set is both analytic and $\mathcal{G}$-analytic. On the other hand, it is easy to see that in a perfectly normal space, every analytic set is strongly analytic.

Proposition $3.1(V=L)$. If $X$ is a space of character $\leqslant \omega_{1}$ such that each subset of $X$ belongs to the $\sigma$-algebra generated by the strongly analytic subsets of $X$, then $X$ is o-discrete.

Proof. Assume that $X$ satisfies the conditions stated in the proposition. Denote by $\mathscr{P}(X)$ (by $\S$ ) the family consisting of all (strongly analytic) subsets of $X$. For any family $\varrho$ of subsets of $X$, denote by $\mathcal{L}_{\sigma}$ (by $\varrho_{\delta}$ ) the family consisting of all countable 
unions (intersections) of sets from $\mathcal{L}$, and let $\sim \mathcal{L}=\{X \sim L: L \in \mathcal{L}\}$. Let $\delta_{0}=\delta \cup$ $\sim \delta$, and define $\delta_{\alpha}$, for $\alpha<\omega_{1}$, recursively by the formula $\delta_{\alpha}=\mathcal{C}_{\alpha} \cup \sim \mathcal{C}_{\alpha}$, where $e_{\alpha}=\left(\cup_{\beta<\alpha} \delta_{\beta}\right)_{\sigma} \cup\left(\cup_{\beta<\alpha} \delta_{\beta}\right)_{\delta}$. Then $\cup_{\alpha<\omega_{1}} \delta_{\alpha}$ is the $\sigma$-algebra generated by $\delta$; consequently, $\cup_{\alpha<\omega_{1}} \delta_{\alpha}=\mathscr{P}(X)$. It follows by $V=L$ (or just $\mathrm{CH}$ ) and Theorem 9 of [3] that there exists $\rho<\omega_{1}$ such that $\delta_{\rho}=\mathscr{P}(X)$.

The remainder of this proof is carried out in two stages. First we use the topology on $X \times\left(\{\omega\} \cup{ }^{<\omega} \omega\right)$ defined by Hansell in the proof of the theorem in [5] to obtain a space $Y$ such that for each $S \in \delta$, the sets $S \times\{\omega\}$ and $(X \sim S) \times\{\omega\}$ can be separated by open subsets of $Y$. Then we define a set $I \subset{ }^{\rho+1} \omega$ and a topology on $Y \times(\{\omega\} \cup I)$ to obtain a space $Z$ such that for each $S \in \delta_{\rho}$, the sets $S \times\{\omega\} \times\{\omega\}$ and $(X \sim S) \times\{\omega\} \times\{\omega\}$ can be separated by open subsets of $Z$; since $\delta_{\rho}=\mathscr{P}(X)$, the space $Z$ turns out to be normal and, by Fleissner's theorem, collectionwise Hausdorff; finally, collectionwise Hausdorffness of $Z$ implies that $X$ is $\sigma$-discrete.

Let $Y=X \times\left(\{\omega\} \cup{ }^{<\omega} \omega\right)$ and topologize $Y$ as follows. Make all the points of $Y \sim X \times\{\omega\}$ isolated. Let the filter $\mathcal{H}$ on ${ }^{<\omega} \omega$ be defined as in the proof of Theorem 2.10. A neighborhood-base for a point $\langle x, \omega\rangle \in X \times\{\omega\}$ consists of all sets of the form

$$
U(x, g)=\{\langle x, \omega\rangle\} \cup \bigcup_{s \in H}(g(s) \times\{s\}),
$$

where $H \in \mathcal{H}$ and $g$ is a map with domain $H$ and range a set of neighborhoods of $x$ in $X$. As $\chi(X) \leqslant \omega_{1}$, it is easily seen that $\chi(Y) \leqslant \omega_{1}$. Let $S$ be a strongly analytic subset of $X$. We show that $S \times\{\omega\}$ and $(X \sim S) \times\{\omega\}$ can be separated by open subsets of $Y$. Let $G_{s}, s \in{ }^{<\omega} \omega$, be open subsets of $X$ such that $S=\cup_{\phi \in \omega^{\omega}} \cap_{n \in \omega} G_{\phi \mid n}$ $=\cup_{\phi \in{ }^{\omega} \omega} \cap_{n \in \omega} \bar{G}_{\phi \mid n}$. The proof of Lemma 2 of [5] shows that we can choose the sets $G_{s}$ so that for all $\phi, \psi \in{ }^{\omega} \omega$ and $n \in \omega, G_{\phi \mid n+1} \subset G_{\phi \mid n}$ and, if $\phi \leqslant \psi$, then $G_{\phi \mid n} \subset G_{\psi \mid n}$. For each $x \in X$, let $H_{x}=\left\{s \in{ }^{<\omega} \omega: x \in G_{s}\right\}$ and $J_{x}=\left\{s \in{ }^{<\omega} \omega: x \notin\right.$ $\left.\vec{G}_{s}\right\}$; note that if $x \in S$, then $H_{x} \in \mathcal{K}$ and if $x \in X \sim S$, then $J_{x} \in \mathcal{H}$. For each $s \in{ }^{<\omega} \omega$, let $g(s)=G_{s}$ and $f(s)=X \sim \bar{G}_{s}$. Let $V=\cup_{x \in S} U\left(x, g \mid H_{x}\right)$ and $W=$ $\cup_{x \in X \sim S} U\left(x, f \mid J_{x}\right)$. Then $V$ and $W$ are open subsets of $Y$ containing $S \times\{\omega\}$, respectively. It is easy to see that $V \cap W=\varnothing$.

Let $I$ be the set of all step-functions in ${ }^{\rho+1} \omega$ (i.e., $f \in I$ iff $f \in{ }^{\rho+1} \omega$ and there are ordinals $0=\alpha_{1}<\cdots<\alpha_{n}=\rho+1$, for some $n \in \omega$, such that $f$ is constant on each of the intervals $\left[\alpha_{i}, \alpha_{i+1}[\subset \rho+1)\right.$. Note that $|I|=\omega$. We use induction on $\alpha$ to define a filter-base $\cup_{\alpha<\rho} \varepsilon_{\alpha}$ on $I$. Let

$$
\mathcal{E}_{0}=\{\{f \in I: f(0) \geqslant n\}: n<\omega\} .
$$

If $\varepsilon_{\beta}$ has been defined for each $\beta<\alpha \leqslant \rho$, then $\varepsilon_{\alpha}$ consists of all sets of the form $\bigcup_{k>n}\left\{s \in E_{k}: s(\alpha)=k\right\}$, where $n \in \omega$ and $E_{k} \in \cup_{\beta<\alpha} \mathscr{E}_{\beta}$ for each $k \geqslant n$. Note that for all $\beta<\alpha \leqslant \rho, \mathcal{E}_{\beta} \subset \mathcal{E}_{\alpha}$; consequently, $\cup_{\alpha \leqslant \rho} \mathcal{E}_{\alpha}=\mathcal{E}_{\rho}$. Each of the families $\varepsilon_{\alpha}$ is closed under finite intersections: this holds trivially for $\alpha=0$; if it holds for each $\alpha<\gamma$, and if $n, m \in \omega$ and $E_{k}, H_{i} \in \cup_{\alpha<\gamma} \mathcal{E}_{\alpha}$ for $k \geqslant n$ and $i \geqslant m$, then

$$
\begin{aligned}
\bigcup_{k \geqslant n}\left\{s \in E_{k}: s(\gamma)=k\right\} & \cap \bigcup_{i \geqslant m}\left\{s \in H_{i}: s(\gamma)=i\right\} \\
= & \bigcup_{j \geq \max (n, m)}\left\{s \in E_{j} \cap H_{j}: s(\gamma)=j\right\} \in \mathcal{E}_{\gamma}
\end{aligned}
$$


so that $\varepsilon_{\gamma}$ is closed under finite intersections. In particular, $\mathcal{E}_{\rho}$ is closed under finite intersections. Each $\mathcal{E}_{\alpha}$ consists of nonempty sets. To see this, we use induction on $\alpha$ to show that for each $\alpha \leqslant \rho$, and for all $f \in I$ and $E \in \mathcal{E}_{\alpha}$, there exists $g \in E$ such that $g|\{\beta: \alpha<\beta \leqslant \rho\}=f|\{\beta: \alpha<\beta \leqslant \rho\}$. The statement holds trivially for $\alpha=0$; assume it holds for each $\alpha<\gamma$. Let $f \in I$ and $E \in \mathcal{E}_{\gamma}$. Let $n \in \omega$ and $E_{k} \in \cup_{\alpha<\gamma} \mathcal{E}_{\alpha}$, for $k \geqslant n$, be such that $E=\cup_{k \geqslant n}\left\{s \in E_{k}: s(\gamma)=k\right\}$. Define $f^{\prime} \in{ }^{\rho+1} \omega$ by setting $f^{\prime}(\gamma)=n$ and $f^{\prime}(\alpha)=f(\alpha)$ for each $\alpha \neq \gamma$; note that $f^{\prime} \in I$. Let $\delta<\gamma$ be such that $E_{n} \in \mathcal{E}_{\delta}$. By the induction assumption, there exists $g \in E_{n}$ such that

$$
g\left|\{\beta: \delta<\beta \leqslant \rho\}=f^{\prime}\right|\{\beta: \delta \leqslant \beta \leqslant \rho\} .
$$

Clearly, $g \in E$ and $g|\{\beta: \gamma<\beta \leqslant \rho\}=f|\{\beta: \gamma<\beta \leqslant \rho\}$; this completes the induction. It follows that $\mathcal{E}_{\rho}$ consists of nonempty sets; hence $\mathcal{E}_{\rho}$ is a filter-base.

Let $Z=Y \times(\{\omega\} \cup I)$. Topologize $Z$ as follows. Make all the points of $Z \sim$ $X \times\{\omega\} \times\{\omega\}$ isolated. A neighborhood-base for a point $\langle x, \omega, \omega\rangle \in X \times\{\omega\} \times$ $\{\omega\}$ consists of all sets of the form

$$
V(x, g)=\{\langle x, \omega, \omega\rangle\} \cup \bigcup_{f \in E}(\varphi(f) \times\{f\}),
$$

where $E \in \varepsilon_{\rho}$ and $\varphi$ is a map with domain $E$ and range a set of neighborhoods of $\langle x, \omega\rangle$ in $Y$. Using that $\chi(Y) \leqslant \omega_{1}$, we easily see that $\chi(Z) \leqslant \omega_{1}$. Note that $X \times\{\omega\} \times\{\omega\}$ is a closed and discrete subset of $Z$ with discrete complement: hence, to show that $Z$ is normal, it suffices to show that for each $S \in \mathscr{P}(X)$, the sets $S \times\{\omega\} \times\{\omega\}$ and $(X \sim S) \times\{\omega\} \times\{\omega\}$ can be separated by open subsets of $Z$. Call an open subset $G$ of $Z \alpha$-open, where $\alpha \leqslant \rho$, provided that for each $\langle x, \omega, \omega\rangle \in$ $G \cap X \times\{\omega\} \times\{\omega\}$, there exists a neighborhood $V(x, \varphi)$ of $\langle x, \omega, \omega\rangle$ contained in $G$ such that $\operatorname{dom}(\varphi) \in \varepsilon_{\alpha}$. For each $\alpha \leqslant \rho$, denote by $\ell_{\alpha}$ the family consisting of all sets $R \subset X$ such that the sets $R \times\{\omega\} \times\{\omega\}$ and $(X \sim R) \times\{\omega\} \times\{\omega\}$ can be separated by $\alpha$-open subsets of $Z$; note that $R_{\alpha}$ is closed under complementation, and because of the corresponding property of $\varepsilon_{\alpha}, \Re_{\alpha}$ is closed under finite intersections. We use induction on $\alpha$ to show that, for each $\alpha \leqslant \rho, S_{\alpha} \subset \ell_{\alpha}$. For each $S \in \Omega$, the sets $S \times\{\omega\}$ and $(X \sim S) \times\{\omega\}$ can be separated by open sets in $Y$; from this it follows that $\varsigma_{0} \subset \ell_{0}$. Let $\alpha$ be such that $0<\alpha \leqslant \rho$ and, for each $\beta<\alpha$, $s_{\beta} \subset \Re_{\beta}$. To show that $s_{\alpha} \subset \Re_{\alpha}$, recall that $s_{\alpha}=e_{\alpha} \cup \sim e_{\alpha}$, where $e_{\alpha}=$ $\left(\cup_{\beta<\alpha} S_{\beta}\right)_{\sigma} \cup\left(\cup_{\beta<\alpha} S_{\beta}\right)_{\delta}$; since $\mathscr{O}_{\beta}$ is an algebra of sets for each $\beta \leqslant \alpha$, the inclusion $S_{\alpha} \subset: R$ follows once we show that for any decreasing sequence $\left\langle S_{n}\right\rangle_{n<\omega}$ of sets from $\cup_{\beta<\alpha} s_{\beta}$, the set $\bigcap_{n<\omega} S_{n}$ belongs to $Q_{h}$. Let $S_{n}, n<\omega$, be members of $\cup_{\beta<\alpha} S_{\beta}$ such that for each $n<\omega, S_{n+1} \subset S_{n}$. For each $n<\omega, S_{n} \in \cup_{\beta<\alpha}$ (Rh ${ }_{\beta}$ and hence there are maps $\varphi_{x, n}$, with domain a member of $\cup_{\beta<\alpha} \bar{\sigma}_{\beta}$ and range a set of neighborhoods of $\langle x, \omega\rangle$ in $Y$, for each $x \in X$, such that for all $x \in S_{n}$ and $y \in X \sim S_{n}, V\left(x, \varphi_{x, n}\right) \cap V\left(y, \varphi_{y, n}\right)=\varnothing$. Define the maps $\varphi_{x}, x \in X$, as follows. Let $x \in X$. If $x \in \cap_{n<\omega} S_{n}$, set $n(x)=0$ and if $x \in X \sim \bigcap_{n<\omega} S_{n}$, let $n(x)<\omega$ be such that $x \notin S_{n(x)}$. Let $E_{x}=\cup_{k \geqslant n(x)}\left\{f \in \operatorname{dom}\left(\varphi_{x, k}\right): f(\alpha)=k\right\}$, and note that $E_{x} \in \varepsilon_{\alpha}$. We make $E_{x}$ the domain of $\varphi_{x}$, and for each $f \in E_{x}$, we let $\varphi_{x}(f)$ be the neighborhood $\varphi_{x, f(\alpha)}(f)$ of $\langle x, \omega\rangle$ in $Y$. Clearly, $\cup\left\{V\left(x, \varphi_{*}\right): x \in \cap_{n<\omega} S_{n}\right\}$ and 
$\cup\left\{V\left(x, \varphi_{x}\right): x \in X \sim \cap_{n<\omega} S_{n}\right\}$ are $\alpha$-open subsets of $Z$ containing $\left(\cap_{n<\omega} S_{n}\right) \times$ $\{\omega\} \times\{\omega\}$ and $\left(X \sim \cap_{n<\omega} S_{n}\right) \times\{\omega\} \times\{\omega\}$, respectively. To see that these sets are disjoint, assume on the contrary that there are $x \in \bigcap_{n<\omega} S_{n}$ and $y \in X \sim$ $\cap_{n<\omega} S_{n}$ such that $V\left(x, \varphi_{x}\right) \cap V\left(y, \varphi_{y}\right) \neq \varnothing$. Then there exists $f \in \operatorname{dom}\left(\varphi_{x}\right) \cap$ $\operatorname{dom}\left(\varphi_{v}\right)$ such that $\varphi_{x}(f) \cap \varphi_{v}(f) \neq \varnothing$. Let $k=f(\alpha)$. Since $f \in \operatorname{dom}\left(\varphi_{1}\right)$, we have $k \geqslant n(y)$ and hence $y \in X \sim S_{k}$. It follows that $V\left(x, \varphi_{x, k}\right) \cap V\left(y, \varphi_{1, k}\right)=\varnothing$ and further, since $f \in \operatorname{dom}\left(\varphi_{x, h}\right) \cap \operatorname{dom}\left(\varphi_{1, h}\right)$, that $\varphi_{x, k}(f) \cap \varphi_{1, k}(f)=\varnothing$; however, this is a contradiction, since $\varphi_{x, k}(f)=\varphi_{x}(f)$ and $\varphi_{y, k}(f)=\varphi_{y}(f)$. We have shown that $\cap_{n<\omega} S_{n} \in \mathscr{G}_{\alpha}$. This completes the proof of the inductive step. Since $S_{p} \subset \Re_{p}$ and $s_{p}=\varphi(X)$, we have proved that $Z$ is normal.

By the theorem in [2], $Z$ is collectionwise Hausdorff. Consequently, we can find mutually disjoint basic neighborhoods $V\left(x, \varphi_{x}\right)$ for the points $\langle x, \omega, \omega\rangle$ of the closed discrete set $X \times\{\omega\} \times\{\omega\}$. For each $x \in X$, let $f_{x} \in \operatorname{dom}\left(\varphi_{x}\right)$, and choose a basic neighborhood $U\left(x, g_{x}\right)$ contained in the neighborhood $\varphi_{x}\left(f_{x}\right)$ of $\langle x, \omega\rangle$ in $Y$, and let $s_{x} \in \operatorname{dom}\left(g_{x}\right)$; note that $g_{x}\left(s_{x}\right)$ is a neighborhood of $x$ in $X$. For all $f \in I$ and $s \in{ }^{<\omega} \omega$, let $D_{f, s}=\left\{x \in X: f_{x}=f\right.$ and $\left.s_{x}=s\right\}$; note that $D_{f, s}$ is a discrete subspace of $X$ since for all distinct $x$ and $y$ in $D_{f, s}, g_{x}\left(s_{x}\right) \cap g_{y}\left(s_{v}\right)=\varnothing$. Since $\left|I \times{ }^{\sim \omega} \omega\right|=\omega$, it follows that $X$ is $\sigma$-discrete.

COROLlaRY $3.2(V=L)$. If $X$ is a perfectly normal space of character $\leqslant \omega_{1}$ such that each subset of $X$ belongs to the o-algebra generated by the analytic subsets of $X$, then $X$ is $F_{\sigma}$-discrete.

Since each metrizable space is perfectly normal and has character $\leqslant \omega_{1}$, Corollary 3.2 and Proposition 2.2 of [7], give the following result.

COROllary $3.3(V=L)$. If $X$ is a $\sigma$-space such that each subset of $X$ belongs to the $\sigma$-algebra generated by the analytic subsets of $X$, then $X$ is $F_{\sigma}$-discrete.

\section{REFERENCES}

1. A. V. Arhangel'skiī and S. J. Nedev. Some remarks on semi-metrizable spaces and their subspaces, C. R. Acad. Bulgare Sci. 31 (1978), no. 5, 499-500.

2. W. G. Fleissner, Normal Moore spaces in the constructible universe, Proc. Amer. Math. Soc. 46 (1974). 294-298.

3. D. H. Fremlin. R. W. Hansell and H. J. K. Junnila, Borel functions of hounded class, Trans. Amer. Math. Soc. (to appear).

4. J. Gerlits and J. Juhász, On left-separated compact spaces, Comment. Math. Univ. Carolinae 19 (1978), 53-62.

5. R. W. Hansell, Some consequences of $(V=L)$ in the theory of analytic sets, Proc. Amer. Math. Soc. 80 (1980). 311-319.

6. H. J. K. Junnila, Neighbornets, Pacific J. Math. 76 (1978), 83-108.

7. On o-spaces and pseudometrizable spaces, Topology Proc. 4(1979), 121-132.

8. G. M. Reed, On normality and countable paracompactness, Fund. Math. 110 (1980), 145-152.

Department of Mathematics, Kossuth University, Debrecen Pf. 12, 4010 Hungary

Department of Mathematics, University of Helsinki, Hallituskatu 15, Helsinki 10, Finland 